\title{
EXPRESSÃO DA COX-2 E CD105 NO CÂNCER DE MAMA E SOBREVIDA LIVRE DE DOENÇA
}

Melina Grudzinski*, Eduardo Cambruzzi, Eduardo lahude, Ricardo francalacci Savaris, José luiz Pedrini, Cláudio Galleano Zettler

Trabalho realizado na Fundação Faculdade Federal de Ciências Médicas de Porto Alegre - Laboratório de Anatomia Patológica e Citopatologia Pós-Graduação, Porto Alegre, RS

\author{
*Correspondência: \\ Rua Coronel Corte Real, \\ 955/202 \\ Cep: $91610-830$ \\ Porto Alegre, RS \\ Tel. (51) 3321-2303 \\ melinag@terra.com.br
}

\begin{abstract}
RESUMO
OBjetivos. Correlacionar a presença de recidiva local de câncer de mama com a expressão de CDI05 em carcinomas primários de mama, e a expressão da ciclooxigenase-2 nos carcinomas primários de mama e nos respectivos linfonodos axilares.

Métodos. Estudo com uma coorte histórica de 72 mulheres entre 29 e 67 anos com diagnóstico de carcinoma ductal infiltrante de mama, estadio II, tipo histológico não especial com seus linfonodos axilares respectivos, que tiveram diagnóstico e tratamento cirúrgico no Hospital Nossa Senhora da Conceição, no período de $200 \mathrm{I}$ a 2002. Análise imunoistoquímica do CDI05 e COX-2 no tumor primário, da COX-2 nos linfonodos axilares e da recidiva local.

Resultados. Das 72 mulheres analisadas com tumores primários, 40 tinham linfonodos axilares positivos e 32 eram negativos; para cada tumor primário, foi escolhido apenas um linfonodo axilar. O grau histológico dos tumores foi I $(n=4)$, II ( $n=4 I)$ e III ( $n=27)$. Quinze pacientes apresentaram recidiva local em um período médio de 26 meses (IC 95\% 24-28). A presença da COX-2 nos tumores primários foi verificada em 52 casos, e a presença de CDI05 em 34 casos, mas não foram considerados fatores prognósticos independentes para recidiva ( $p=0,203$ e $p=0,145$, respectivamente). A sobrevida para pacientes com expressão da COX-2 em linfonodos axilares (metastáticos ou não metastáticos) foi de 19 meses, contra 28,3 meses para pacientes COX-2 negativa $(p<0,000 \mathrm{I})$.
\end{abstract}

Conclusão. A expressão positiva da COX-2 em linfonodos axilares (positivos ou não) parece ser um fator prognóstico independente para sobrevida livre de doença.

UnITERMOS: Câncer de mama. Ciclooxigenase 2. CDI05.

\section{InTRODUÇÃO}

O câncer de mama é a segunda neoplasia maligna mais incidente nas mulheres do Brasil, tendo elevada mortalidade'. As células tumorais são drenadas pela via linfática e o comprometimento dos linfonodos axilares é considerado o mais importante dos fatores prognósticos, juntamente com as dimensões do tumor, no momento da cirurgia ${ }^{2}$. A identificação de novos marcadores que possam predizer o comportamento dos linfonodos e dos tumores é importante no câncer de mama devido à variabilidade na progressão da doença ${ }^{3}$.

As ciclooxigenases (COX) são potenciais marcadores para os casos de câncer de mama ${ }^{4,5}$. As COX são responsáveis pela síntese de prostaglandinas a partir da liberação de ácido araquidônico pelas fosfolipases da membrana celular. A isoforma COX-I é a enzima essencial, expressa na maioria das células e tecidos, responsável pela produção das prostaglandinas PGE2 e PGI2, as quais são importantes em muitos processos fisiológicos, como agregação plaquetária, regulação da corrente sangüínea renal e proteção da mucosa gástricab. A COX-2 é a forma induzível, mitogênica e é expressa em resposta ao processo inflamatório e a outros estímulos fisiológicos, incluindo fatores de crescimento ${ }^{7}$. Há evidências apoiando o papel da COX-2 na carcinogênese $e^{8-10}$, incluindo tumores sólidos de mama ${ }^{11-14}$. A expressão da COX-2 não está confinada ao componente epitelial do tumor; existe uma relação linear significativa entre a neovascularização e a expressão aumentada da COX-29,15.

Outro potencial marcador para o câncer de mama é a endoglina $a^{4.5}$. A endoglina (CD I05) é uma glicoproteína homodimérica de membrana que se liga ao fator de crescimento transformador ßI (TGF-ßI) e TGF$B 3^{16}$ e é preferencialmente expressa em vasos em proliferação durante a angiogênese ${ }^{5}$. Há estudos demonstrando que a expressão aumentada de CD 105, em uma área de neoangiogênese de um tumor de mama, está relacionada com um mau prognóstico ${ }^{17}$. A determinação do índice angiogênico parece ser um fator prognóstico nos carcinomas de mama ${ }^{18,19}$

Com base nesses achados, tomamos como hipótese que a expressão da COX-2 também poderia estar alterada nos linfonodos axilares e poderia ser mais um fator prognóstico a ser usado para o câncer de mama. Após revisão da literatura (Pubmed 1966-2005), não fomos capazes de identificar nenhum trabalho que verificasse essa relação. $O$ objetivo do presente estudo é comparar as pacientes que têm uma expressão aumentada de CDI05 e da COX-2 em tumores primários de câncer de mama, e as que têm expressão aumentada da COX-2 nos linfonodos axilares quanto à incidência de recidiva local do tumor em dois anos. 
GRUDZINSKI M ET AL.

\section{Métodos}

\section{Pacientes}

Este estudo caracteriza-se por uma coorte retrospectiva. Foram incluídas na coorte inicial todas as pacientes com o diagnóstico de carcinoma ductal invasivo de mama, não especial, primário, histologicamente confirmado, submetidas a excisão adequada (margens cirúrgicas livres), com seus respectivos linfonodos axilares, sem tratamento antes da cirurgia, sem metástases à distância, estadio II, durante os anos 200 I e 2002, operadas no Hospital Nossa Senhora da Conceição, em Porto Alegre, Rio Grande do Sul. Todas as pacientes realizaram quimioterapia adjuvante e, nos casos selecionados, radioterapia, conforme as orientações do Instituto Nacional de Câncer (Inca). Os casos de neoplasias do estroma mamário, metástases à distância, demais tipos de carcinoma de mama e pacientes debilitadas por outras doenças associadas foram excluídos. A coleta dos dados a respeito da sobrevida livre de recorrência local foi realizada por meio dos registros médicos. A sobrevida livre da doença foi definida como o período entre o diagnóstico do tumor primário e a recorrência local. A recorrência local foi definida como qualquer recidiva do câncer na mama ipsilateral, na parede torácica ou na pele após terapia inicial ${ }^{20}$. As biópsias das lesões suspeitas de recidiva foram realizadas pelo mesmo mastologista (ULP), por meio da pistola para biópsia (trucut) ou biópsia excisional.

\section{Materiais}

As peças cirúrgicas foram obtidas após mastectomia ou setorectomia, com remoção completa dos três níveis da cadeia de linfonodos axilares. O material era fixado em formalina e encaminhado para o Serviço de Patologia do Hospital Nossa Senhora da Conceição. Os linfonodos foram analisados em conjunto, sem identificação do nível axilar. O estadiamento utilizado foi TNM (tumor/linfonodos/metástases) de acordo com a International Union Against Cancer21, e o grau histológico definido de acordo com a Organização Mundial da Saúde 22 .

Foram analisadas 72 pacientes com tumores primários e seus 72 linfonodos axilares representativos (40 metastáticos e 32 não metastáticos). Para cada tumor primário foram realizados três cortes do bloco de parafina, sendo um para hematoxilina e eosina ( $\mathrm{HE}$ ) para confirmação diagnóstica, e os demais para análise imunoistoquímica da COX-2 e CD 105. Cada paciente teve a sua axila esvaziada, e o linfonodo axilar mais representativo foi escolhido para a análise. A seleção do linfonodo foi baseada na lâmina com a melhor coloração (HE) e com a maior metástase. Para análise dos linfonodos axilares foram realizados dois cortes em bloco de parafina, um deles para confirmação diagnóstica $(\mathrm{HE})$ e o outro para análise imunoistoquímica da COX-2. A análise imunoistoquímica do CDI05 foi realizada apenas no tumor primário das 72 pacientes.

\section{Imunoistoquímica}

Os cortes foram realizados com $3 \mu \mathrm{m}$ de espessura e estendidos em lâminas com 3-aminopropil-trietoxi-silano (Sigma Chemical Co, St Louis, MO USA). As lâminas foram desparafinadas em estufa a $55^{\circ} \mathrm{C}$ e depois removidas em três banhos de xilol, a $60^{\circ} \mathrm{C}, 55^{\circ} \mathrm{C}$ e à temperatura ambiente, durante 60, 30 e 20 minutos, respectivamente. A seguir, os cortes foram hidratados em concentrações decrescentes de etanol, seguidos por lavagens em água corrente. A recuperação antigênica das

\section{Figura 1 - Sobrevida livre de doença em pacientes de acordo com a} expressão da COX-2 nos linfonodos axilares

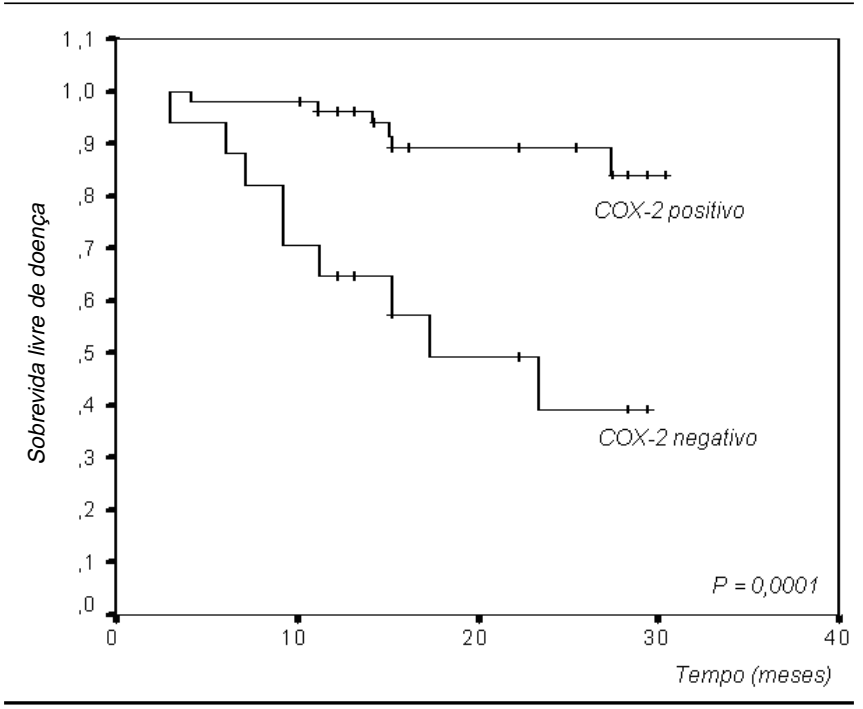

amostras foi obtida com calor úmido sob pressão em solução de tampão citrato $10 \mathrm{mM} / \mathrm{pH}$ 6,0. Posteriormente, foi realizado 0 bloqueio da peroxidase tecidual endógena por meio de três passagens de 15 minutos em solução de peróxido de hidrogênio 10\%. Logo após, as lâminas foram submetidas a soro de cabra para bloqueio dos sítios não-espeć́ficos. Após três banhos com solução tampão de fosfato (PBS), procedeu-se a incubação dos anticorpos primários, anti-COX-2 (IgG, Dako Lab., New York, USA) e anti-CDI05 (Novocastra Lab., Balliol Business Park West, London, UK). Os anticorpos foram diluídos ( I:50, ambos) em PBS pH 7,2 com I\% de albumina sérica bovina, em câmara úmida a $4^{\circ} \mathrm{C}$ por 24 horas. Depois de três lavagens em PBS, os cortes foram incubados com o anticorpo secundário de cabra anticamundongo biotinilado, conforme as orientações do fabricante (Dako). Os cortes foram novamente lavados três vezes durante 5 minutos em PBS. Logo depois, foi aplicado o complexo estreptoavidina-biotina peroxidase de acordo com as orientações do fabricante (Dako), durante 30 minutos em câmara úmida a $37^{\circ} \mathrm{C}$. Para a revelação da reação, foi utilizada a solução de diaminobenzidina (DAB - Dako) em PBS (I $5 \mu$ g de diaminobenzidina para cada $25 \mathrm{ml} \mathrm{de} \mathrm{PBS} \mathrm{pH}$ $7,2)$. Após a revelaçãa, as lâminas foram lavadas em água corrente e coradas em hematoxilina. Os cortes foram desidratados em álcool absoluto por cinco vezes durante 2 minutos em cada etapa e banhados em xilol. As lâminas foram montadas em resina do tipo Entellan (Merck, Darmstadt, Alemanha).

Todas as etapas foram feitas com a utilização de controles positivos externos, tecidos de carcinoma de mama com padrão de revelação já conhecido para COX-2 e CD I05, e o controle negativo foi realizado em casos de carcinoma ductal invasivo, sem a colocação dos anticorpos primários. As lâminas foram avaliadas por dois pesquisadores (EC, MG) de forma independente e cegada, sob microscopia óptica. Os resultados foram agrupados em negativo e positivo. A positividade para expressão da COX-2 foi avaliada pela fórmula do HSCORE (HSCORE $=\Sigma P i)$, onde 


\begin{tabular}{lcc}
\hline & Tabela 1 - Características & \\
\hline & $\mathbf{n}$ & $(\%)$ \\
\cline { 2 - 3 } Câncer de mama primário & 72 & 100 \\
Tratamento conservador* & 42 & 58,3 \\
Mastectomia & 30 & 41,7 \\
\hline Tratamento complementar & & \\
Radioterapia & 48 & 66,6 \\
Quimioterapia & 72 & 100 \\
Com recidiva local em até 28 meses & 15 & 20,8 \\
COX-2 positivo/negativo & $51 / 21$ & $71 / 29$ \\
CDI05 positivo/negativo & $34 / 38$ & $47,2 / 52,8$ \\
Linfonodos & 72 & 100 \\
Metastáticos/não metastáticos & $40 / 32$ & $55,5 / 44,6$ \\
COX-2 positivo/negativo & $18 / 54$ & $25 / 75$ \\
Metastáticos e COX-2 positivo/negativo & $17 / 23$ & $42,5 / 57,5$ \\
Não metastáticose COX-2 positivo/negativo & $\mid / 31$ & $3,1 / 96,9$ \\
\hline Grau histológico & & \\
I & 4 & 5,5 \\
II & 41 & 57 \\
III & 27 & 37,5 \\
\hline *T & &
\end{tabular}

*Teste exato de Fisher

Tabela 2 - Presença de recidiva local de acordo com as características do tumor primário e do linfonodo axilar

\begin{tabular}{|c|c|c|c|}
\hline \multirow{3}{*}{ Tumor primário } & \multicolumn{2}{|c|}{ Recidiva } & \multirow[t]{3}{*}{$p^{*}$} \\
\hline & Sim & Não & \\
\hline & $\overline{n(\%)}$ & $\overline{n(\%)}$ & \\
\hline COX-2 positivo & $13(18)$ & $38(53)$ & \\
\hline COX-2negativo & $2(3)$ & $19(26)$ & 0,203 \\
\hline CDI05 positivo & $10(14)$ & $24(33,3)$ & \\
\hline CDI05 negativo & $5(7)$ & $33(45,8)$ & 0,145 \\
\hline \multicolumn{4}{|l|}{ Linfonodo axilar } \\
\hline COX-2 positivo & $10(14)$ & $8(11)$ & \\
\hline COX-2negativo & $5(7)$ & $49(68)$ & $<0,000 \mid$ \\
\hline Metastático & $13(18)$ & $27(38)$ & \\
\hline Nãometastático & $2(3)$ & $30(42)$ & 0,008 \\
\hline Met*** COX-2 positivo & $9(22,5)$ & $8(20)$ & \\
\hline Met + COX-2 negativo & $4(10)$ & $19(47,5)$ & 0,03 \\
\hline
\end{tabular}

* Teste exato de Fisher

*** Met: metastático

$\mathrm{P}=$ porcentagem de células com citoplasma amarronzado em I0 campos de grande aumento, e i= grau de intensidade da coloração (variação de 0 = ausência de coloração, $\mid$ = fraca, 2 =média e 3 =forte). $O$ produto de cada intensidade era somado, o que dava um HSCORE que variava entre 0 e 300 . Um escore $\geq 100$ era considerado positivo conforme descrito na literatura ${ }^{15}$.

A positividade para CDI 05 foi feita nas áreas de hot spot. Cada vaso com coloração amarronzada foi considerado um vaso individual. $O$ valor final foi o resultado da média do número de vasos corados em quatro campos de grande aumento. $O$ ponto de corte utilizado para positivo foi $\geq 15$ vasos conforme descrito na literatura ${ }^{18}$. $O$ controle positivo para CDI05 foi o endotélio dos vasos presentes na lâmina parte tumoral.

\section{Análise estatística e tamanho da amostra}

O cálculo do tamanho da amostra foi baseado conforme dados da literatura ${ }^{23}$. Para tanto, foi considerada a recidiva de tumor local em cinco anos. Os casos com câncer de mama com $\geq 80 \%$ de expressão para COX-2 tinham $60 \%$ de recidiva em cinco anos, enquanto que os casos com $<75 \%$ de expressão tinham $20 \%$ de recidiva em cinco anos $^{24}$, com erro $\alpha=0,05$ e erro $\beta=0,2$. Utilizando os dados da literatura, deveríamos ter 54 casos no total. Os dados foram processados e analisados com o auxílio do programa SPSS versão I0.0 (Chicago, IL, USA). Foi utilizado o teste exato de Fisher para análise das variáveis e dos fatores prognósticos no tumor e linfonodo, e análise de Kaplan Meyer para avaliação do tempo de sobrevida livre de doença.

Esse estudo foi submetido e aprovado pelo Comitê de Ética em Pesquisa do Hospital Nossa Senhora da Conceição, relatório $n^{\circ} 048 / 03$.

\section{Resultados}

A média de idade das pacientes diagnosticadas com carcinoma ductal invasivo de mama foi de 55,5 anos ( $D P \pm 12,51$ ); sendo que 27 pacientes possuíam idade inferior ou igual a 50 anos no momento do diagnóstico. Todas as pacientes foram tratadas conforme os protocolos do Instituto Nacional de Câncer. As pacientes se encontravam em um mesmo estadiamento inicial no momento da análise (inception cohort). Todas as cirurgias conservadoras foram complementadas por radioterapia. Nas mastectomias, a radioterapia foi aplicada somente nos casos de mais de quatro linfonodos positivos. As demais características das 72 pacientes com câncer primário de mama e seus respectivos linfonodos estão demonstradas na Tabela I. Das 72 pacientes, I5 (20,8\%) apresentaram recidiva local em um período médio de 26 meses (IC 95\%: 24-28 meses). A mediana do tempo de seguimento foi de 16 meses.

\section{Expressão da COX -2 e CD105 no tumor}

A expressão da COX-2 foi avaliada em 72 pacientes com carcinoma ductal invasivo de mama e foi restrita às células tumorais em todas as amostras positivas; fibroblastos e células endoteliais adjacentes não demonstraram a presença da COX-2. Cinqüenta e uma pacientes tiveram expressão positiva para a COX-2 (7|\%). Do total de I 5 pacientes com recidiva local, 13 apresentaram a expressão da COX-2 no tumor primário (recidiva local em 24,4 meses), e duas não expressaram COX-2 (recidiva local em 27,7 meses). A presença da COX-2 no tumor primário não pode ser considerada com fator prognóstico independente para sobrevida livre de doença, ( $p=0,203$; Tabela 2).

Das 72 pacientes com tumores primários de mama, 34 casos foram considerados CDI05 positivo. Dos 15 casos que tiveram recorrência local, 10 eram CD I 05 positivo, e 5 eram CD 05 negativo. A expressão de CDI05 não apresentou significância estatística como fator prognóstico ( $p=0,145$; Tabela 2).

\section{Expressão da COX-2 nos linfonodos e sobrevida livre de doença}

O esvaziamento axilar foi realizado nas 72 pacientes. De cada paciente, o linfonodo mais representativo foi obtido. Dos 72 
linfonodos, 40 eram metastáticos e 32 não eram metastáticos. Dezoito linfonodos (25\%) foram considerados COX-2 positivo, e 54 (75\%) eram COX-2 negativo. As pacientes com linfonodos COX-2, positivo independentemente de serem metastáticos ou não, tiveram recidiva local em 19, I meses. Dentre as 54 pacientes com linfonodos (metastáticos ou não) COX-2 negativo, cinco tiveram recidiva em 28,3 meses, sendo esta uma diferença significativa $(p<0,000$ I) (Tabela 2).

Das 40 pacientes com linfonodos axilares metastáticos, I3 apresentaram recorrência local, sendo que, destas, nove apresentavam linfonodos com a expressão da COX-2 e oito não $(p=0,03)$. Dentre as 32 pacientes com linfonodos axilares não metastáticos, duas tiveram recidiva, e apenas uma tinha linfonodo com a expressão para COX-2 $(p=0,06)$. A presença da COX-2 em todos os linfonodos axilares foi igualmente considerada como fator prognóstico independente, com maior força para sobrevida livre de doença $(p=0,00 \mathrm{I}$ e $p=0,00 \mathrm{I}$; respectivamente). Da mesma forma, existe uma associação entre a expressão de COX2 e metástase no linfonodo $(p<0,000$ I)

\section{Discussão}

Este estudo tentou verificar se as pacientes que têm uma expressão aumentada de CDI05 e da COX-2 em tumores primários de câncer de mama, e as que têm expressão aumentada da COX-2 nos linfonodos axilares têm uma incidência maior de recidiva de tumor local em dois anos. Para responder esta pergunta, o cálculo inicial do tamanho da amostra seria de 54 pacientes. Todavia, levando em consideração as diferenças na prevalência da COX-2 na população em estudo, e mantendo o erro alfa em 0,05 e o beta em 0,20, verificamos que seriam necessários no mínimo 18 casos COX-2 positivo e 18 casos COX-2 negativo. Esse número foi atingido, permitindo que os resultados mantivessem o poder estatístico pré-estabelecido.

\section{Expressão de CD105}

A capacidade de invasão do estroma é uma característica fundamental na progressão tumoral, mas não necessariamente a primeira etapa; em muitos casos, a angiogênese tem início prévio. Em câncer de mama, assim como em outros tipos de câncer, o aumento da densidade microvascular tem sido relatado como um fator prognóstico independente para a sobrevida das pacientes, relacionado com altas taxas metastáticas ${ }^{16,18,25,26}$. Como era de se esperar, o CDI05 só foi demonstrado no endotélio de vasos tumorais ${ }^{26}$, todavia, esse marcador não se mostrou preditor para a recidiva local. Os nossos resultados não estão de acordo com os relatados por Kumar et al. ${ }^{26}$ e Dales et al. ${ }^{18}$, que demonstraram que a presença aumentada de CDI05 ( $\geq 15$ vasos) estava relacionada com um risco maior de metástases em pacientes com linfonodos negativos. A diferença entre os nossos resultados e os demonstrados por esses autores pode ser explicada pelo tempo de seguimento. $O$ nosso estudo teve um menor tempo de seguimento, não dando chance para o aparecimento do desfecho. No trabalho original de Dales et al., não havia diferença na curva de sobrevida livre de doença até os 24 meses de seguimento, sendo que a diferença começava a se tornar mais marcante aos 96 meses $^{18}$.

\section{Expressão da COX-2 nos tumores primários}

A expressão da COX-2 nas amostras obtidas de tumores primários de pacientes com câncer de mama não mostrou relação com o prognóstico de recidiva local. Nossos resultados reafirmam os conflitos existentes sobre o significado da expressão da proteína e o prognóstico, assim como a freqüência da expressão da COX-2 no câncer de mama. As explicações para essas diferenças ainda têm de ser descobertas 11,14 .

\section{Expressão da COX -2 em linfonodos}

Embora o mecanismo de atuação da COX-2 na tumorogênese ainda não esteja estabelecido, a associação entre eles tem sido relatada em diversos estudos, ${ }^{9,24}$. A angiogênese é responsável pelo suprimento de nutrientes e oxigênio às novas células, além de proporcionar a disseminação vascular do tumor. O nosso estudo, assim como o de Costa et al. ${ }^{14}$, demonstrou a expressão da COX2 nos casos com carcinoma ductal infiltrante de mama, mas não em tecido mamário normal, apesar da diferença nos critérios para positividade. Para nosso conhecimento, após extensa revisão de literatura (Pubmed 1966-2005), não encontramos outro trabalho que tivesse analisado a expressão da ciclooxigenase-2 em linfonodos axilares de pacientes com câncer de mama e o relacionasse à sobrevida livre de doença. A presença da COX-2 em linfonodos axilares ( 17 metastáticos e um não metastático) determinou uma diferença significativa na sobrevida livre de doença ( 19 meses contra 28,3 meses) para pacientes com linfonodos com expressão negativa para COX-2.

A expressão aumentada do marcador em linfonodos foi considerada como fator prognóstico independente para recidiva, tendo uma associação com a presença de linfonodos metastáticos ou não. A relevância desse achado pode estar na precocidade da diferença (26 meses). A evidência do envolvimento da ciclooxigenase- 2 com a angiogênese sugere que as pacientes com linfonodos axilares metastáticos associados à expressão da COX-2 venham a ter um pior prognóstico. Sabendo que a axila é considerada um dos mais importantes fatores prognósticos para recorrência da doençç,27,28, os resultados obtidos a partir deste trabalho sugerem que a expressão da COX-2 em linfonodos axilares, sejam eles metastáticos ou não, possa se tornar igualmente importante como fator prognóstico para recorrência, visto que pacientes com a expressão positiva do marcador no linfonodo apresentaram recidiva precoce. Isso poderá abrir nossas perspectivas no tratamento com drogas específicas para COX-2. Em termos clínicos e práticos, os médicos devem estar atentos para a possibilidade de começar a usar inibidores da COX-2 no tratamento ou prevenção do câncer de mama. Além disso, este trabalho abre a possibilidade da análise multivariada da expressão de COX-2 em linfonodos axilares em conjunto com os outros marcadores para o câncer de mama. A COX-2 pode vir a fazer parte da rotina de marcadores para a sobrevida livre de doença, assim como é o receptor de estrogênio nos tumores de mama. 


\section{CONCLUSÃo}

A expressão positiva da COX-2 em linfonodos axilares com ou sem metástase parece ser um fator prognóstico independente para sobrevida livre de doença

\section{Conflito de interesse: não há}

\section{Summary}

\section{COX-2 AND CD105 EXPRESSION IN BREAST CANCER AND DISEASE- FREE SURVIVAL}

OBIECTNE. To verify the expression of CD 105 in primary breast cancer, and the expression of cyclooxygenase-2 in primary breast cancer and in the respective axillary lymph nodes.

METHODS. Seventy two women from 18 to 80 years of age, with a diagnosis of Ductal Infiltrative Breast Cancer, stage II, histologicaltype non special, with their respective axillary lymph nodes were submitted to surgical treatmentat the "HospitalNossa Senhora da Conceição" between 200I and 2002. Immunohistochemicalanalyses of CD I 05 in the primary breast cancer and of COX 2 in the local axillary lymph node were comparedforlocal recurrence.

RESULTS. Of the 72 patients with primary tumors, 40 had positive axillary lymph nodes, while 32 were negative. For each primary tumor, only one lymph node was analyzed. . Fifteen patients had local recurrence after 26 months (CI 95\% 24-28). Presence of COX-2 in the primary tumors was verified in 52 cases, and presence of $C D 105$ in 34 cases. These independent prognostic factors were not correlated to local recurrence $P=0.203 \mathrm{eP}=0.145$, respectively). The period free of local recurrence for patients with positive expression of COX2 in axillary lymph nodes (with metastasis or not) was of 19 months, while patients with negative expression had a 28.3 months long period free of local recurrence.

CONCLUSION. The positive expression of COX-2 in axillarylymph nodes (either positive or negative) seems to be an independent prognostic factor for local recurrence. [Rev Assoc Med Bras 2006; 52(4): 275-80]

KEY WORDs: Breast cancer. Cyclooxygenase 2. CDI05.

\section{RefERÊnCIAS}

I. Brasil.Ministério da Saúde. Estimativas da incidência e mortalidade por câncer no Brasil. Instituto Nacional do Câncer - INCA 2003. Available from: http://www.inca.gov.br/conteudo_view.asp?id=336

2. Fitzgibbons PL, Page DL, Weaver D, Thor AD, Allred DC, Clark GM, et al. Prognostic factors in breast cancer. College of American Pathologists Consensus Statement 1999. Arch Pathol Lab Med 2000; 1 24:966-78.

3. Gradishar WJ. The future of breast cancer: the role of prognostic factors. Breast Cancer Res Treat 2005;89(Suppl I):SI7-S26.

4. Vainio H, Morgan G. Cyclo-oxygenase 2 and breast cancer prevention. Non-steroidal anti-inflammatory agents are worth testing in breast cancer. BMJ 1998:317:828.

5. Dales JP, Garcia S, Bonnier P, Duffaud F, Andrac-Meyer L, Ramuz O, et al. CD 05 expression is a marker of high metastatic risk and poor outcome in breast carcinomas. Correlations between immunohistochemical

analysis and long-term follow-up in a series of 929 patients. Am J Clin Pathol 2003; | 1 9:374-80.

6. Simmons DL, Botting RM, Hla T. Cyclooxygenase isozymes: the biology of prostaglandin synthesis and inhibition. Pharmacol Rev 2004;56:387-437.

7. Simon LS. Role and regulation of cyclooxygenase-2 during inflammation. Am J Med 1999; 106:37S-42S.

8. Hussain T, Gupta S, Mukhtar H, Smith WL, DeWitt DL, Garavito RM. Cyclooxygenase-2 and prostate carcinogenesis cyclooxygenases: structural, cellular, and molecular biology. Cancer Lett 2003; 191: 125-35.

9. Li G, Yang T, Yan J. Cyclooxygenase-2 increased the angiogenic and metastatic potential of tumor cells. Biochem Biophys Res Commun 2002;299:886-90

10. Subbaramaiah K, Dannenberg AJ. Cyclooxygenase 2: a molecular target for cancer prevention and treatment. Trends Pharmacol Sci 2003;24:96-102.

II. Howe LR, Subbaramaiah K, Brown AM, Dannenberg AJ. Cyclooxygenase-2: a target for the prevention and treatment of breast cancer. Endocr Relat Cancer 200 1;8:97- I I 4.

12. Hwang D, Scollard D, Byrne J, Levine E. Expression of cyclooxygenase- I and cyclooxygenase-2 in human breast cancer. J Natl Cancer Inst 1998;90:455-60.

13. Subbaramaiah K, Norton L, Gerald W, Dannenberg AJ. Cyclooxygenase-2 is overexpressed in HER-2/neu-positive breast cancer: evidence for involvement of AP-I and PEA3. J Biol Chem 2002; 277: / 8649-57.

14. Costa C, Soares R, Reis-Filho JS, Leitao D, Amendoeira I, Schmitt FC. Cyclo-oxygenase 2 expression is associated with angiogenesis and lymph node metastasis in human breast cancer. J Clin Pathol 2002;55:429-34.

I5. Davies G, Salter J, Hills M, Martin LA, Sacks N, Dowsett M. Correlation between cyclooxygenase-2 expression and angiogenesis in human breast cancer. Clin Cancer Res 2003;9:265I-6.

16. Duff SE, Li C, Garland JM, Kumar S. CD 105 is important for angiogenesis: evidence and potential applications. FASEB J 2003; 17:984-92.

17. Guidi AJ, Berry DA, Broadwater G, Perloff M, Norton L, Barcos MP, et al. Association of angiogenesis in lymph node metastases with outcome of breast cancer. J Natl Cancer Inst 2000;92:486-92.

18. Dales JP, Garcia S, Carpentier S, Andrac L, Ramuz O, Lavaut MN, et al. Long-term prognostic significance of neoangiogenesis in breast carcinomas: comparison of Tie-2/Tek, CDI05, and CD3I immunocytochemical expression. Hum Pathol 2004;35: 176-83.

19. Dales JP, Garcia S, Andrac L, Carpentier S, Ramuz O, Lavaut MN, et al. Prognostic significance of angiogenesis evaluated by CDI05 expression compared to CD31 in 905 breast carcinomas: correlation with long-term patient outcome. Int J Oncol 2004;24: I 197-204.

20. Recht AC, Troyan SL, Sadowsky NL. Local-regional recurrence after mastectomy or breast-conserving therapy. In: Harris JR, Lippman ME, Morrow M, Osborne CK, editors. Disease of the breast. 3rd ed. Philadelphia: Lippincott Williams \& Wilkins; 2000.p.731-4.

21. Sobin LH, Wittekind Ch. TNM Classification of Malignant Tumours (UICC). 6th ed. New York: Wiley-Liss; 2002.

22. World Health Organization. Pathology and genetics of tumours of the breast and female genital organs. Oxford: Oxford University Press; 2003.

23. Browner WS, Newman TB, Cummings SR, Hulley SB. Estimando o tamanho de amostra e o poder estatístico: pontos básicos. In: Hulley SB, Cummings SR, Browner WS, Grady D, Hearst N, Newman TB, editors. Delineando a pesquisa clínica, uma abordagem epidemiológica. 2nd ed. Porto Alegre: Artmed; 2003. p.83- 104. 
GrUDZINSKI M ET AL.

24. O'Connor JK, Avent J, Lee RJ, Fischbach J, Gaffney DK. Cyclooxygenase2 expression correlates with diminished survival in invasive breast cancer treated with mastectomy and radiotherapy. Int J Radiat Oncol Biol Phys 2004;58: 1034-40.

25. Dales JP, Garcia S, Carpentier S, Andrac L, Ramuz O, Lavaut MN, et al. Prediction of metastasis risk (I I year follow-up) using VEGF-RI, VEGFR2, Tie-2/Tek and CDI05 expression in breast cancer $(n=905) . B r ~ J$ Cancer 2004;90:1216-21.

26. Kumar S, Ghellal A, Li C, Byrne G, Haboubi N, Wang JM, et al. Breast carcinoma: vascular density determined using CD 105 antibody correlates with tumor prognosis. Cancer Res 1999;59:856-61.
27. Carter CL, Allen C, Henson DE. Relation of tumor size, lymph node status, and survival in 24,740 breast cancer cases. Cancer 1989;63:181-7.

28. Han S, Yun IJ, Noh DY, Choe KJ, Song SY, Chi JG. Abnormal expression of four novel molecular markers represents a highly aggressive phenotype in breast cancer. Immunohistochemical assay of p53, nm23, erbB-2, and cathepsin D protein. J Surg Oncol 1997;65:22-7.

Artigo recebido: 27/06/05 Aceito para publicação: 18/1 1/05

Nota do editor: No trabalho "Fatores associados à interrupção de tratamento anti-retroviral em adultos com AIDS. Rio Grande do Norte, Brasil, 1999 - 2002", publicado na revista n- 2 - volume 52 - março/abril 2006, página 86 a 92, a ordem correta dos autores é Ana Maria de Brito; Célia Landmann Szwarcwald; Euclides Ayres de Castilho. 